\title{
Mass Spectrometric Study on Sodium Ion Induced Central Nucleotide Deletion in the Gas Phase
}

\author{
Helga Dögg Flosadóttir, Kristmann Gíslason, Snorri Thor Sigurdsson, Oddur Ingólfsson
}

Science Institute and University of Iceland, Department of Chemistry, Dunhagi 3, 107 Reykjavík, Iceland

\begin{abstract}
We report a mass spectrometric study on sodium ion induced central nucleotide deletion from protonated oligonucleotides (ONTs) and the concurrent recombination of the terminal nucleotides. To shed some light on the mechanism behind this intriguing fragmentation channel, we have studied the metastable decay of a number of different protonated hexameric and octameric oligonucleotides with 0-6 and 0-8 of their exchangeable protons replaced with sodium ions, respectively. In selected cases, we have also studied the further fragmentation of the parent ions after initial base loss. Our findings are concurrent with a reaction mechanism where the initial step is the elimination of a protonated, high proton affinity (PA) base from the center of the ONTs. This is followed by an elimination of a (next neighbour) nucleotide that contains a second high PA base and the concurrent recombination of the terminal nucleotides. To our knowledge, such central nucleotide deletion in the gas phase has only been reported in one previous study (Flosadóttir et al., J. Am. Soc. Mass Spectrom 20:689-696, 2009), and this is the first systematic approach to understand the mechanism behind this channel.
\end{abstract}

Key words: Metastable decay, Sodium adducts, Oligonucleotide fragmentation, Central nucleotide deletion, Fragmentation mechanism, DNA strand folding, DNA secondary structure

\section{Introduction}

ince the DNA $\beta$-helix was characterized by Watson and $\curvearrowright$ Crick, many new naturally occurring secondary structures of single, double, and triple stranded DNA have also been characterized, such as hairpins and other cruciforms [1,2]. Although the double stranded helix is the most common secondary structure of DNA, the cruciforms and smaller structures have been found to be very important in gene expression and replication processes, as well as in other biological functions [3]. Different DNA structures and their functions are therefore of great scientific interest.

Electronic supplementary material The online version of this article (doi:10.1007/s13361-011-0335-1) contains supplementary material, which is available to authorized users.

Correspondence to: Oddur Ingólfsson; e-mail: odduring@hi.is
Metal cations have been shown to participate in the formation of many DNA secondary structures [4-8]. In such secondary structures, in aqueous solutions, the interaction between DNA and sodium is similar to an organometallic complex, where the DNA ligand sites are arranged around the sodium [9, 10]. The DNA ligand sites that bond to sodium ions are the nucleobase ketone groups and the nonbridging oxygen atoms of the phosphodiesters [5]. Due to solvation of DNA in aqueous solution, however, the sodium cations are not localized at specific coordination sites, but fluctuate between sites. In the absence of a solvent, as found in the gas phase, the sodium-phosphate and the sodium base connections are stronger [11]. In aqueous media, the cation can be located between the oxygen atoms of the phosphodiesters, the nucleobases and water molecules with a preferred coordination number of six. Similarly, in the gas phase, the sodium cation can coordinate with more than one group of the oligonucleotide (ONT) [5]. This may in turn 
result in stabilization of a specific conformation of the ONT strand $[5,12]$. An example of such stabilization was shown by Martínez et al. [10] using molecular dynamics simulations, which showed a $(\mathrm{pdT})_{8}$ single strand fold in the presence of eight sodium ions. In this fold, the three terminal bases on each side are stacked, forcing the central bases $\left(\mathrm{B}_{4}\right.$ and $\mathrm{B}_{5}$ ) out from the main turn.

Recently, we have studied the metastable decay of the protonated and deprotonated hexameric ONTs 5'-d(TTXYTT) $(\mathrm{X} \neq \mathrm{Y}$ are $\mathrm{C}, \mathrm{G}$, or $\mathrm{A})$. The protonated and deprotonated parent ions were found to behave similar with respect to the loss of a high proton affinity (PA) base and backbone cleavage [13, 14]. However, for the protonated ONTs, an intriguing, new major fragmentation channel was observed. This channel led to the same $\mathrm{m} / \mathrm{z}$ ratio for all the hexameric ONTs studied $(\mathrm{m} / \mathrm{z} 1332+$ n22), generally opened up after the exchange of two protons against sodium ions, reached a maxima in intensity when five protons were exchanged against sodium ions and was abruptly "turned-off" when the sixth proton was exchanged against a sodium ion. This fragment-mass corresponds to the elimination of a central base and the second central nucleotide and recombination of the two terminal d(TT) chains, hence the formation of a fragment containing all four thymidines and a deoxyribose unit that has lost one water molecule. To simplify the discussion, we will refer to this fragment as $\left(\mathrm{R}-\mathrm{H}_{2} \mathrm{O}\right) \mathrm{dT}_{4}$.

To shed some light on the mechanism behind this intriguing fragmentation channel we have studied the metastable decay of the protonated hexameric ONTs 5'-O methyl d(TTGCTT), 5'-O methyl d(TTCGTT), 5'-d(TTGTTT), 5'-d(TTTGTT), 5'-d(TTGGTT), 5'-d(TGCTTT), and 5'-d(TTTGCT) with 0-6 of their exchangeable protons replaced with sodium ions as well as the metastable decay of the two octameric oligonucleotides 5'-d(TTTGCTTT) and 5'-d(TTTCGTTT) with 0-8 of their exchangeable protons replaced with sodium ions. In selected cases we have studied the further fragmentation of the parent ions after initial base loss. The results show that this new fragmentation channel proceeds through a central nucleotide and base elimination that is promoted by the exchange of the phosphodiester protons against sodium ions.

To our knowledge this study, along with our previous report on the hexameric ONTs, is the first reported and experimentally verified central elimination of nucleotides from single stranded oligonucleotides in the gas phase. Such central elimination of single nucleotides or sequences of nucleotides is known to occur in solutions as a deletion mutation, which is a mutation commonly observed in solid tumours [15].

\section{Experimental}

The metastable decay measurements of the protonated oligonucleotides were carried out with a commercial matrix assisted laser desorption/ionisation time of flight mass spectrometer (MALDI-TOF); REFLEX IV (Bruker Daltonics, Bremen, Germany) that is operated in post-source decay (PSD) mode. The instrument and its operation for metastable decay measurements of shorter ONTs have been described in detail before [14], and thus, we only give a short description here. The REFLEX IV is a reflectron type UV-MALDI-TOF equipped with a $400 \mu \mathrm{J} /$ pulse $\mathrm{N}_{2}$-laser operating at $10 \mathrm{~Hz}$ and $337 \mathrm{~nm}$. The experiments were performed in positive ion, pulsed delayed extraction mode with $400 \mathrm{~ns}$ delay time. The total acceleration voltage was $25 \mathrm{kV}$ resulting in about $30 \mu \mathrm{s}$ flight time through the field free linear region (ca. $1.25 \mathrm{~m}$ ) before arrival at the reflectron. The protonated parent ions are mass selected with an ion gate placed about $75 \mathrm{~cm}$ downstream from the acceleration region. The width of the mass gate was set to be $\pm 5 \mathrm{Da}$ in all experiments. After the linear flight, the ions are mass analysed with a grid-less reflectron and detected on a double micro-channel plate detector. The reflectron voltage was stepped down in seven segments to assure for observation of all fragment masses from the precursor mass, down to $m / z 600$. Each segment is the sum of 500 laser shots and is recorded by using the FlexControl ${ }^{\mathrm{TM}}$ software provided by the manufacturer. The segments are combined and calibrated to create one PSD mass spectra, using the FlexAnalysis ${ }^{\mathrm{TM}}$ software, also provided by the manufacturer. In all experiments, the laser power was kept about $10 \%$ above the detection threshold of the respective molecular ions as higher laser power would lead to more extensive in-source decay (ISD) that complicates the parent ion selection for the PSD experiments. Mass accuracy was verified by comparing the measured mass of the precursor and that of the $\mathrm{w}_{2}$ and $\mathrm{w}_{3}$ fragments with the calculated masses.

For sample preparation, $2 \mu \mathrm{L}$ of a $0.1 \mathrm{mM}$ solution of the respective ONTs were mixed with $0.5 \mu \mathrm{L}$ of $0.01 \mathrm{M} \mathrm{NaCl}$ solution to increase the degree of $\mathrm{H}^{+} / \mathrm{Na}^{+}$exchange. This sodium concentration was chosen as higher concentration interfered with the crystallization of the sample spot and lower concentrations gave insufficient signal intensity for ONTs with more than few protons exchanged with sodium ions. The sample solution $(2.5 \mu \mathrm{L})$ was mixed with $2 \mu \mathrm{L}$ of saturated aqueous solution of 2,5-dihydroxybenzoic acid (DHB), spotted on a polished stainless steel sample carrier and allowed to dry in air. Also other matrixes were tested and gave in principle the same results with respect to the PSD.

The two modified hexameric oligonucleotides 5'-O methyl $\mathrm{d}($ TTGCTT) and 5'-O methyl d(TTCGTT) and their native analogues 5'-d(TTGCTT) and 5'-d(TTCGTT), respectively, were synthesized from the phosphoramidites of the native nucleotides with standard protecting groups (Glen Research, Sterling, VA, USA), and the in-house prepared phosphoramidite, 5'-O methyl, 3'-phosphoramidite of thymidine, as previously described [14]. The 5'-O methyl, 3'-phosphoramidite thymidine was synthesized from 5'-O methyl thymidine (Carbosynth, Compton, Berkshire, UK) [16]. Synthesis and purification protocols are provided with the supplementary material. All unmodified oligonucleotides were purchased HPLC purified from TAG Copenhagen A/S (Copenhagen, Denmark) and used as received.

In addition to the PSD measurements for the protonated parent ions we also studied the secondary fragmentation of 
protonated 5'-d(TTGCTT) and 5'-d(TTCGTT) after the loss of the guanine in the in-source decay. These experiments were conducted on an AUTOFLEX TOF/TOF (Bruker Daltonics, Bremen, Germany), which operation is somewhat different from the REFLEX IV, but the principle is the same. For the secondary fragmentation studies we used 3-hydroxy picolinic acid (3-HPA) as matrix. The principal of these measurements (the AUTOFLEX) and the sample preparation are described in supplementary material.

\section{Results and Discussion}

In previous studies [13, 14], we measured the metastable decay of the sodium-free and the sodium adducts of all combinations of the protonated hexameric ONTs 5 '-d(TTXYTT) $(\mathrm{X} \neq \mathrm{Y}$ are $\mathrm{C}, \mathrm{G}$, or $\mathrm{A})$. The most pronounced fragmentation channels observed for the sodium-free hexamers, were the loss of one of the high PA nucleobases, with dominating contribution from the higher PA base, as well as the same base loss followed by a $3^{\prime}-\mathrm{C}-\mathrm{O}$ bond rupture at the base loss site. The 3'-C-O bond rupture results in the formation of two complementary fragment ions that are commonly referred to as $\mathrm{a}_{4}-\mathrm{B}_{4}$ and $\mathrm{w}_{3}$ according to a nomenclature proposed by McLuckey et al. (see Figure 1) [17].

From these, the $\mathrm{a}_{4}-\mathrm{B}_{4}$ fragment dominates when the higher PA base is in the fourth position and $\mathrm{w}_{3}$ dominates when the higher PA base is in the third position. Hence, apparently the $\mathrm{a}_{4}-\mathrm{B}_{4}$ fragments result from initial base loss from the fourth position and the $\mathrm{w}_{3}$ fragments result from initial base loss from the third position [13]. When the exchangeable protons were, one by one, replaced by sodium ions, a gradual quenching of the backbone cleavage was observed, but a new channel leading to the same fragment for all the ONTs, i.e., $\left(\mathrm{R}-\mathrm{H}_{2} \mathrm{O}\right) \mathrm{dT}_{4}$, generally opened up after the exchange of 2-3 protons against sodium ions. The high PA base loss, on the other hand, stayed fairly constant as the first five protons were exchanged against sodium ions.

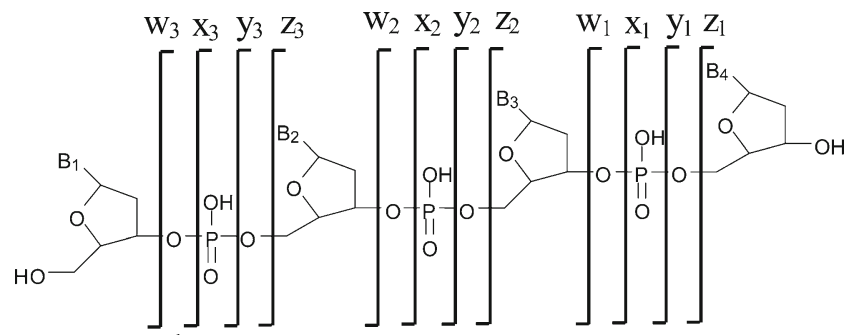

$\begin{array}{llllllllllll}a_{1} & b_{1} & c_{1} & d_{1} & a_{2} & b_{2} & c_{2} & d_{2} & a_{3} & b_{3} & c_{3} & d_{3}\end{array}$

Figure 1. Schematic representation of the nomenclature for single stranded oligonucleotide fragmentation as proposed by McLuckey et al. [17]. Lower case letters denote the bond dissociation site and the charge retention ( $a, b, \ldots 3^{\prime}$-site, $w, x, \ldots$ 5 '-site). Subscript numbers denote the number of nucleotides remaining on the charge retention site. For strand dissociation and base elimination the fragment is written, minus the base (e.g., $a_{2}-B_{2}$ is the $a_{2}$ fragment less $B_{2}$ ). The figure is reproduced from ref. [17]
However, after the sixth proton was exchanged against a sodium ion the base loss was close to quantitatively quenched. This can be rationalized by considering the PA and the alkali metal ion affinity of the DNA nucleobases. The PA is in the order $\mathrm{T}<<\mathrm{A} \approx \mathrm{C}<\mathrm{G}(209.0,224.2,225.9$, and $227.4 \mathrm{kcal} / \mathrm{mol}$, respectively) [18]. The affinity of the nucleobases towards lithium and potassium has been shown to be in the same order, but significantly lower [19]. One can therefore assume that the sodium affinities follow the same order. The sixth sodium thus preferably attaches to the highest PA nucleobase and inhibits its protonation and, thereby, the elimination. The quenching of the strand cleavage with the exchange of the first four protons against sodium ions, on the other hand, indicates that the backbone cleavage is critically dependent on the availability of an proton at one of the phosphodiesters. The fragmentation channel leading to the central nucleotide excision and the recombination of the terminal $\mathrm{d}(\mathrm{TT})$ groups, i.e., the formation of the $\left(\mathrm{R}-\mathrm{H}_{2} \mathrm{O}\right) \mathrm{dT}_{4}$ fragment, behaved very differently compared with the classical backbone cleavage channels. This fragmentation channel, which generally opened up after the exchange of two protons against sodium ions, reached a maxima in intensity when five protons were exchanged against sodium ions. However, similar to the base loss this channel was abruptly "turned-off" when the sixth proton was exchanged against a sodium ion.

Previously, a fragmentation mechanism was suggested for this channel, which involved a nucleophilic attack from the terminal $5^{\prime}-\mathrm{OH}$ to the phosphate in the fourth position [13]. In this case, methylation of the 5'-OH terminal should reduce its nucleophilic character and quench this fragmentation channel. We have thus recorded the PSD spectra of protonated 5'-O methyl d(TTGCTT) and 5'-O methyl d(TTCGTT) with 0-6 protons exchanged against sodium ions. Figure 2 shows these spectra for 5'-O methyl $\mathrm{d}$ (TTGCTT) (the spectra for 5'-O methyl d(TTCGTT) are shown with the supplementary material).

It is clear from Figure 2 that the 5'-O methyl d(TTGCTT) fragments behave just like the previously studied hexameric ONTs. This is true for the backbone cleavage and the base loss and it also forms the same $\left(\mathrm{R}-\mathrm{H}_{2} \mathrm{O}\right) \mathrm{dT}_{4}$ fragment after the exchange of two protons against sodium ions. The only difference is that the fragment mass is shifted to $\mathrm{m} / \mathrm{z} 1346+$ $22 \mathrm{n}$ due to the additional methyl group which remains within the positively charged fragment. This is also the case for 5'-O methyl d(TTCGTT) and shows clearly that the previously proposed nucleophilic attack from the 5'-hydroxyl group is not on the reaction pathway for the formation of this fragment.

To obtain more information on the sequence dependency of the fragmentation mechanism, and the role of the high PA bases, we measured the metastable decay of the protonated ONTs; 5'-d(TTGTTT) and 5'-d(TTTGTT), with 0-6 protons exchanged against sodium ions. The spectra are shown with the supplementary material. For 5'-d(TTGTTT) we only observe $\mathrm{w}_{3}$ fragment formation, showing that the formation of this fragment is critically dependent on the high PA base being in 


\section{$\mathrm{ONa}$ \\ 5'-O methyl d(TTGCTT)}
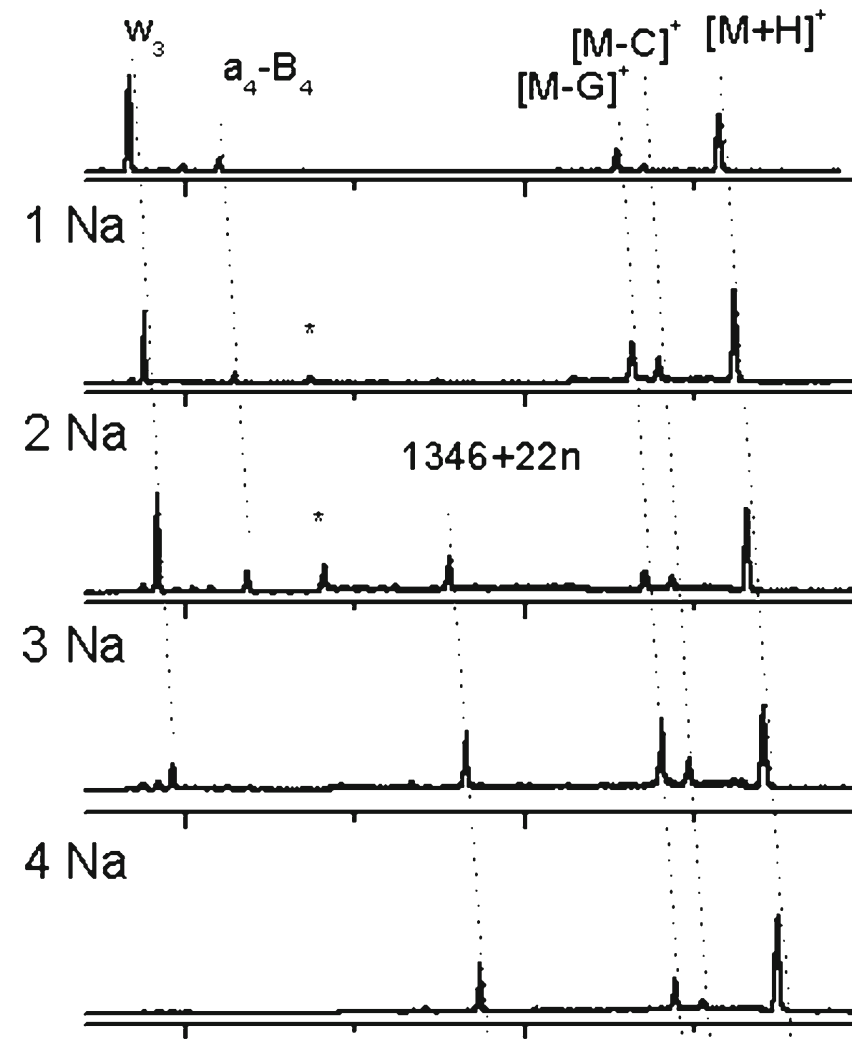

$5 \mathrm{Na}$

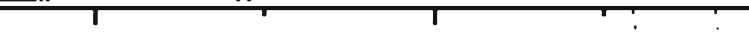

$6 \mathrm{Na}$

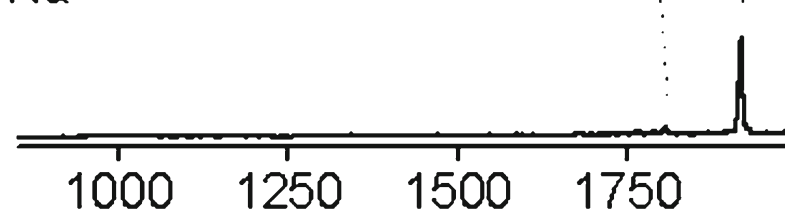

$\mathrm{m} / \mathrm{z}$

Figure 2. PSD spectra of protonated 5'-O methyl d(TTGCTT) with 0-6 protons exchanged against sodium cations. The number of sodium ions is denoted for each spectrum. Sodium ions gradually quench the strand dissociation, while base elimination still prevails. A new channel is observed after an exchange of two protons against sodium ions. The sixth sodium ion closes all fragmentation channels. The peaks marked with asterisks are attributed to artefacts at the junctions of two segments of the mass spectra

the third position. Similarly, we only observe $\mathrm{a}_{4}-\mathrm{B}_{4}$ fragment formation from 5'-d(TTTGTT) showing that its formation is critically dependent on the high PA base being in the fourth position. It is also clear that the relative contribution of the high PA base loss increases as the backbone cleavage $\left(\mathrm{a}_{4}-\mathrm{B}_{4}\right.$ and $\left.\mathrm{w}_{3}\right)$ is quenched. Furthermore, no $\left(\mathrm{R}-\mathrm{H}_{2} \mathrm{O}\right) \mathrm{dT}_{4}$ fragment formation is observed in these spectra, showing clearly that the second high PA base is necessary to open up this fragmentation channel. To further verify the need for two adjacent high PA nucleobases for the formation of $\left(\mathrm{R}-\mathrm{H}_{2} \mathrm{O}\right) \mathrm{dT}_{4}$, we also measured the metastable fragmentation of 5 '-d(TTGGTT). Figure 3 compares line graphs of the relative yield of individual fragments from 5'-d(TTTGTT) and 5'-d(TTGGTT) with 0-6 protons exchanged against sodium ions (the individual spectra are shown with the supplementary material).

Though comparison between segments in the PSD spectra should be taken with some reservation, the trend is clear. From 5'-d(TTGGTT) the formation of $\left(\mathrm{R}-\mathrm{H}_{2} \mathrm{O}\right) \mathrm{dT}_{4}$ increases as the backbone cleavage $\left(\mathrm{a}_{4}-\mathrm{B}_{4}\right.$ and $\left.\mathrm{w}_{3}\right)$ decreases, but the single base-loss yield stays fairly constant. From 5'-d(TTGTTT), on the other hand, the relative yield of single base-loss increases as the backbone cleavage $\left(a_{4}-B_{4}\right.$
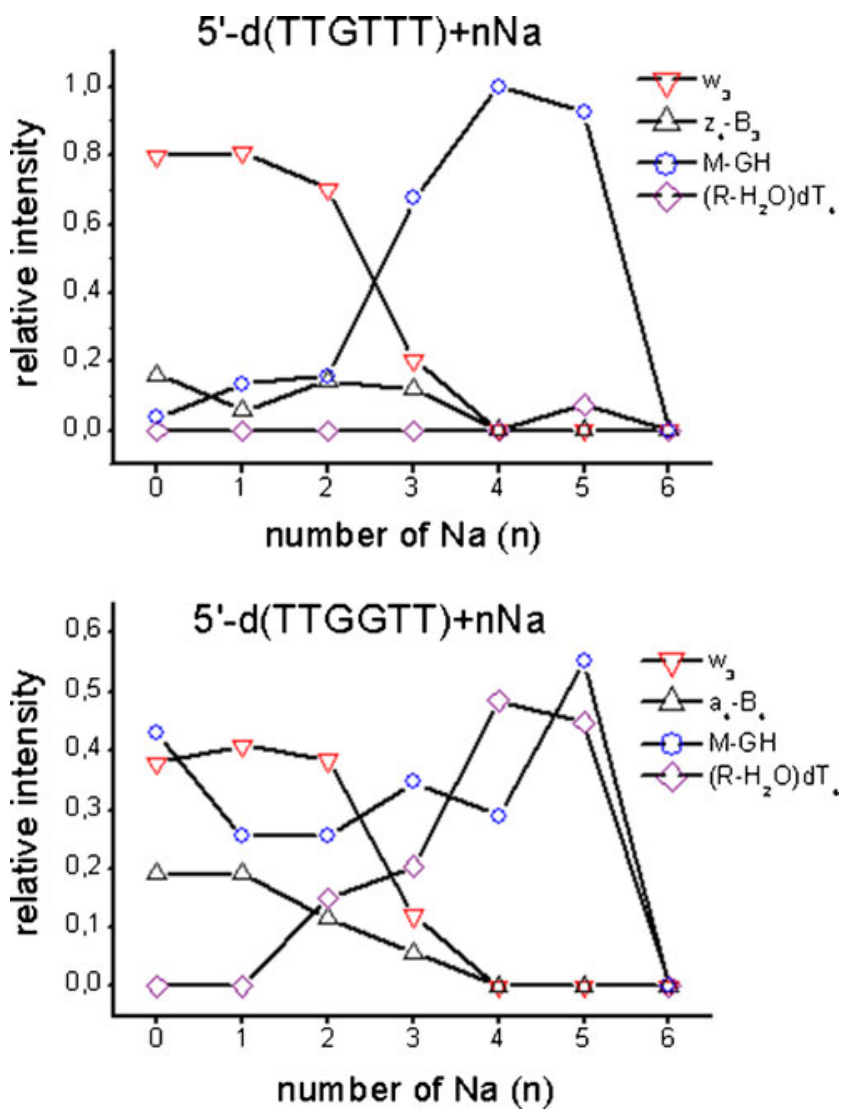

Figure 3. Line graph for the relative yield of individual fragments from 5'-d(TTGTTT) (top) and 5'-d(TTGGTT) (bottom) where 0-6 protons have been exchanged against sodium ions. For the hexameric oligonucleotide containing only one central high PA nucleobase; 5'-d(TTGTT), the relative yield of the base loss increases as the backbone cleavage is quenched. For the hexameric oligonucleotide 5'-d(TTGGTT) the new fragmentation channel leading to $\left(\mathrm{R}-\mathrm{H}_{2} \mathrm{O}\right) \mathrm{dT}_{4}$ is open when $n \geq 2$ and increases as other backbone cleavages are quenched, while the single base loss yield stays fairly constant. Both these fragments are abruptly quenched when $n=6$ 
and $\left.\mathrm{w}_{3}\right)$ decreases. The backbone cleavage and the $\left(\mathrm{R}-\mathrm{H}_{2} \mathrm{O}\right) \mathrm{dT}_{4}$ channels are thus competing in the sense that they both rely on the same fragment ion as precursor. Hence, when the $\mathrm{a}_{4}-\mathrm{B}_{4}$ and $\mathrm{w}_{3}$ channels are quenched, more of the protonated molecular ions that have lost one of the high PA bases are available for $\left(\mathrm{R}-\mathrm{H}_{2} \mathrm{O}\right) \mathrm{dT}_{4}$ formation. The comparison of the line spectra in Figure 3 also confirms that the availability of the second high PA base is crucial for the formation of the $\left(\mathrm{R}-\mathrm{H}_{2} \mathrm{O}\right) \mathrm{dT}_{4}$ fragment.

To further verify that the high PA base loss is the first step in the formation of these fragments, we have studied PSD of protonated [5'-d(TTGCTT) - G] and [5'-d(TTCGTT) - G] formed in the ISD in the MALDI process, before the pulsed ion extraction. PSD spectra of these fragment ions with 0-4 protons exchanged against sodium ions are shown with the supplementary material. Both these fragment ions show further fragmentation in PSD, leading to backbone cleavage as well as $\left(\mathrm{R}-\mathrm{H}_{2} \mathrm{O}\right) \mathrm{dT}_{4}$ formation, and the dependence of these channels on the number of protons exchanged against sodium ions is the same as was observed for the parent ions. Furthermore, from the protonated fragment [5'-d(TTGCTT) - G] we nearly exclusively observe the backbone cleavage through the $\mathrm{w}_{3}$ channel and from [5'-d(TTCGTT) - G], backbone cleavage is nearly exclusively observed through the $\mathrm{a}_{4}-\mathrm{B}_{4}$ channel.

In addition to the 5'-d(TTXYTT) hexamers studied previously, we now also studied the metastable decay of the ONTs 5'-d(TGCTTT) and 5'-d(TTTGCT). Similar to the previously studied 5 '-d(TTXYTT) hexamers, the hexameric oligonucleotides, 5'-d(TGCTTT), and 5'-d(TTTGCT) have two adjacent high PA nucleobases. These are, however, shifted towards the 5'- end and the 3'- end, respectively. The fragmentation channel leading to the central nucleobase and nucleotide excision, as well as concurrent recombination of the terminal nucleotides, is also observed from these ONTs. This channel does, however, not open up until four protons have been exchanged against sodium ions in these ONTs, and the yield is also comparatively low. Interestingly, the fragment formed upon recombination of the terminal nucleotides from these ONTs contains four thymidine units. Hence, from these ONTs also a $\left(\mathrm{R}-\mathrm{H}_{2} \mathrm{O}\right) \mathrm{dT}_{4}$ fragment is formed. This shows that the necessary (sodium induced) base stacking required to bring the terminal thymidines in sufficient proximity for their recombination is also achievable with only one thymidine at one end and three at the other. In this context it is worth mentioning that in the previous study of the protonated 5'-d(TTXYTT) hexamers, also the metastable decay of 5'-d(CTGCTT), 5'-d(TTGCTC), and 5'-d(CTGCTC) was measured [13]. For these ONTs, the central nucleotide deletion was also observed, though to a lesser extent, which is most likely due to less favorable interaction in the conformation enabling this channel. Interestingly, however, the resulting tetramer fragments contained the terminal C's. Hence, either the $\mathrm{C}$ base(s) participated with the $\mathrm{T}$ bases in the sodium induced base stacking, or two terminal T's are sufficient to support the structural stability of the folded hexamer, necessary

\section{$5^{\prime}-d$ (TTTGCTTT)}

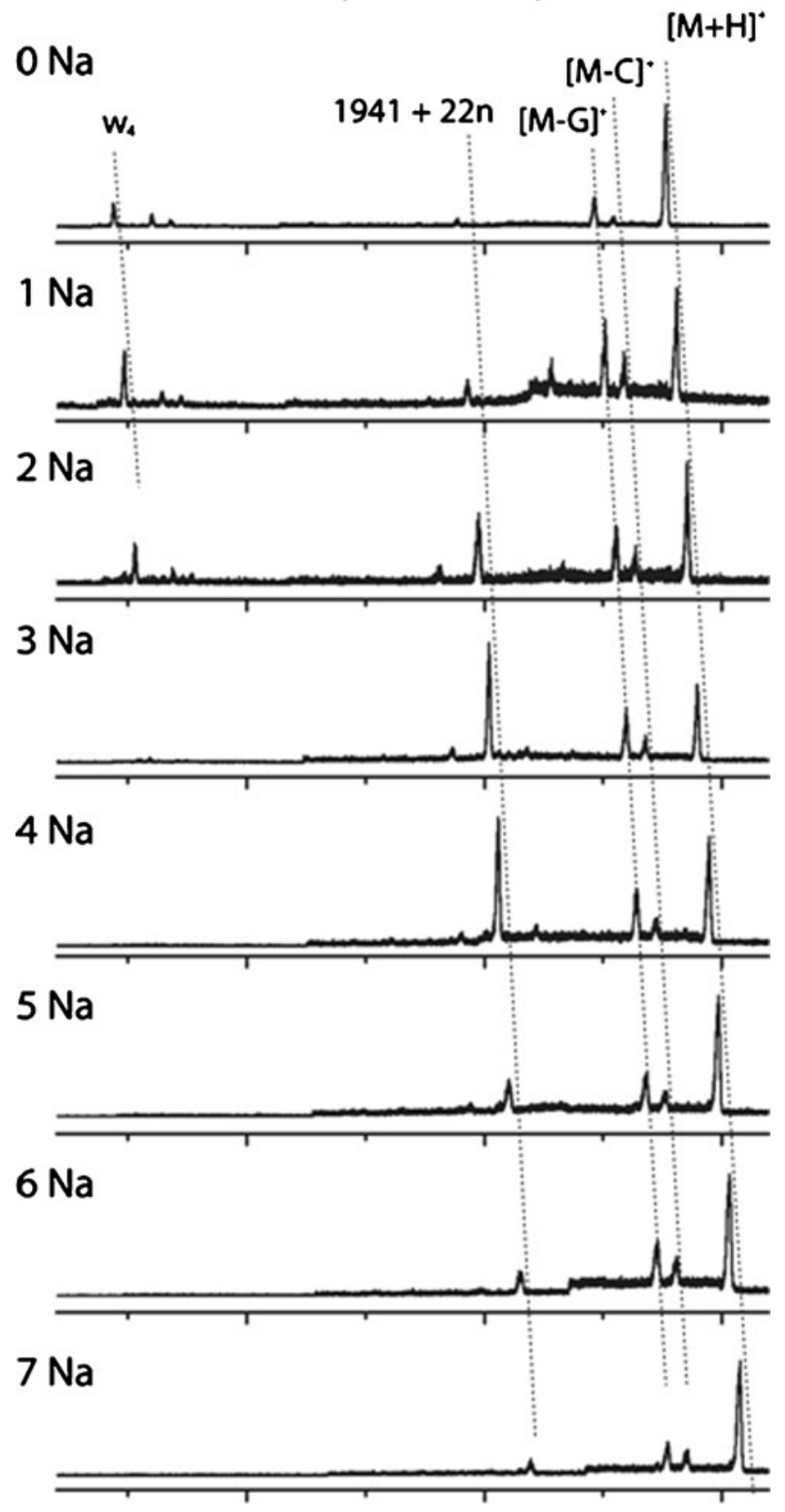

$8 \mathrm{Na}$

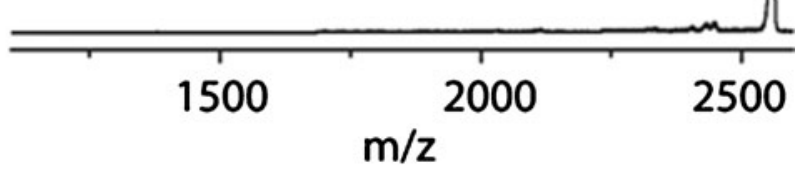

Figure 4. PSD spectra of protonated 5 '-d(TTTGCTTT) with 0-8 protons exchanged against sodium ions. The number of sodium ions is denoted for each spectrum. Sodium ions gradually quench the strand dissociation, while base elimination prevails. The new channel is observed already before ion exchange. The eighth sodium ion closes all fragmentation channels 
for the combination of the terminal groups. In fact, calculations performed by Baker et al. [5] for dinucleotides, including $\mathrm{d}(\mathrm{TT})$, have shown that the energetically most favorable conformation is where the bases are stacked and that this conformation is further stabilized through sodium counter ions.

To further verify the role of the strand conformation in enabling the central nucleotide deletion, and more specific, the role of $\mathrm{H}^{+} / \mathrm{Na}^{+}$exchange in acquiring the preferred conformation, we have measured the metastable decay of the octameric ONTs; 5'-d(TTTGCTTT) and 5'-d(TTTCGTTT) with 0-8 protons exchanged against sodium ions. The elongation of the ONT chain increases its flexibility and changes the relative position of the terminal groups with respect to the cleavage site, and should thus affect the fragmentation. Figure 4 shows the PSD spectra of 5'-d(TTTGCTTT) with 0-8 protons exchanged against sodium ions (the spectra for 5 -d(TTTCGTTT) are shown with the supplementary material). These octamers show qualitatively the same behaviour as the hexameric ONTs 5'-d (TTXYTT). The main fragmentation channels for the sodiumfree octamers are the high PA nucleobase loss and the backbone cleavage leading to $\mathrm{a}_{5}-\mathrm{B}_{5}$ and $\mathrm{w}_{4}$. The $\mathrm{a}-\mathrm{B}$ and w channels are all quantitatively quenched after the exchange of four protons against sodium ions but the base loss prevails until seven protons have been exchanged against sodium ions. However, the excision of one of the central nucleobases and one of the central nucleosides in conjunction with the recombination of the terminal d(TTT) chains is already operative, though to a limited extent, without the $\mathrm{H}^{+} / \mathrm{Na}^{+}$ exchange. With the exchange of two protons against sodium ions, this channel is already the dominating fragmentation path for both 5'-d(TTTGCTTT) and 5'-d(TTTCGTTT). This shows that the longer $\mathrm{T}$ terminals, and thereby the overall length of the ONTs, stabilizes the reactive conformation necessary for the central nucleotide deletion. This is in good agreement with the theoretical study performed by Martínez et al. [10], which showed that non-consecutive base stacking between the terminal $\mathrm{T}$ chains is induced by sodium adduct formation and that such stacking is favored for longer chains. The authors showed that the oligonucleotide $(\mathrm{pdT})_{8}$, with eight sodium counter ions, undergoes a central folding to attain the energetically most favorable conformation. In this conformation, the terminal nucleobases 1,2 , and 3 stack with the terminal
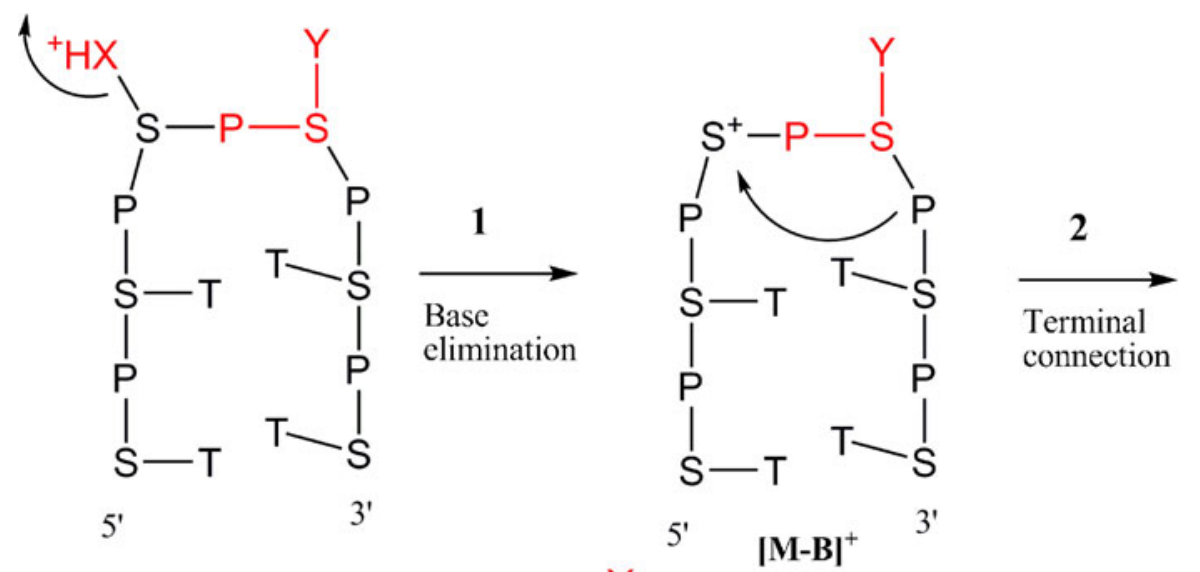

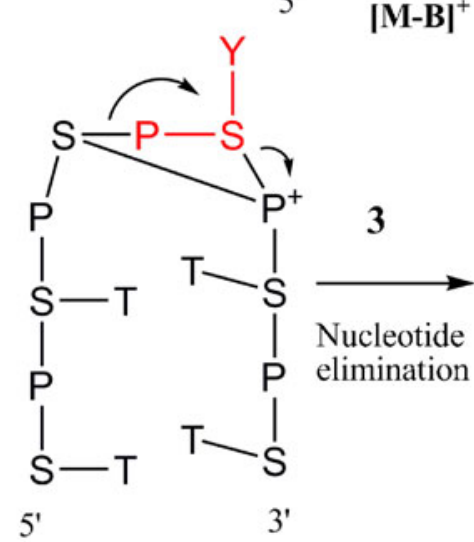

$[\mathbf{M}-\mathbf{B}]^{+}$<smiles>[3H]PS[SH+]SPS[3H]</smiles>

$\left(\mathrm{R}-\mathrm{H}_{2} \mathrm{O}\right) \mathrm{dT}_{4}$

Figure 5. A schematic view of the fragmentation channel, which eliminates a central nucleotide and one central nucleobase, and combines the two TT terminals. Strand folding and base stacking induced by sodium cations enables this fragmentation channel by decreasing the distance between non-consecutive nucleotides. The fragmentation channel is initiated by a neutral base elimination and a further nucleophilic attack from a phosphate group to the resulting, positively charged sugar. The second high PA nucleotide is then removed to form $\left(\mathrm{R}-\mathrm{H}_{2} \mathrm{O}\right) \mathrm{dT}_{4}$ 
bases 6,7 , and 8 , respectively [10]. This structure strains the single stranded DNA, isolates the central nucleobases 4 and 5, and reduces the distances between the nucleotides 3 and 6 surrounding the center.

In our study, it is clear from the recombination of the terminal groups alone that a folding of the ONTs is a prerequisite for the central nucleotide deletion. However, the role of the sodium ions in the folding process is less clear and we can not exclude that the correlation between the extent of the $\left(\mathrm{R}-\mathrm{H}_{2} \mathrm{O}\right) \mathrm{dT}_{4}$ formation and the degree of $\mathrm{H}^{+} / \mathrm{Na}^{+}$ exchange, is partly due to the quenching of the backbone cleavage channels.

In summary, we conclude from our study on the central nucleotide deletion in conjunction with a recombination of the terminal thymidines that (1) the 5'-OH terminal group does not participate in the fragmentation mechanism, (2) the first step is an elimination of a protonated high PA base, (3) two adjacent high PA bases are required to enable this channel, (4) the reactive conformation, assuring the proximity of the terminal groups requires folding of the ONTs, which is most likely achieved (stabilized) up on $\mathrm{H}^{+} / \mathrm{Na}^{+}$ exchange.

Figure 5 shows schematically a proposed mechanism for the neutral base and nucleotide loss and the concurrent recombination of the terminal T-groups.

In Figure 6, a proposed mechanism that we find to be concurrent with our observations is given in more detail for initial base loss from the third position. In the proposed mechanism the first step is the elimination of one of the high PA bases, enabled by its protonation. In this step, the neutral base is eliminated and the charge remains at its sugar moiety. A nucleophilic attack from a nonadjacent phospho-
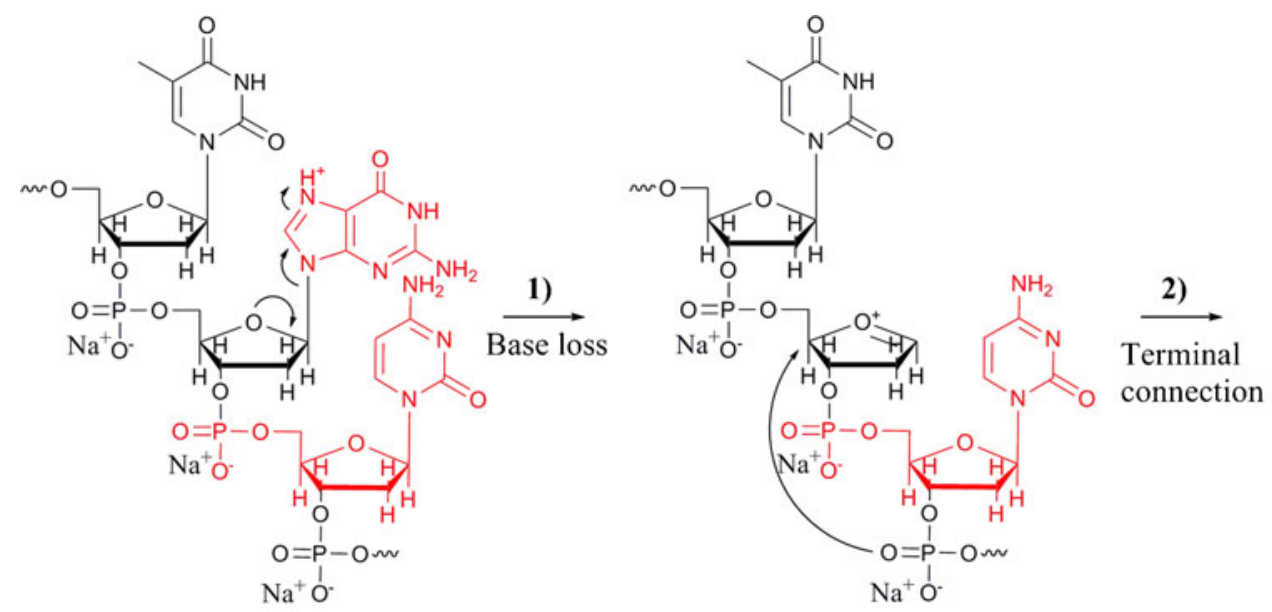

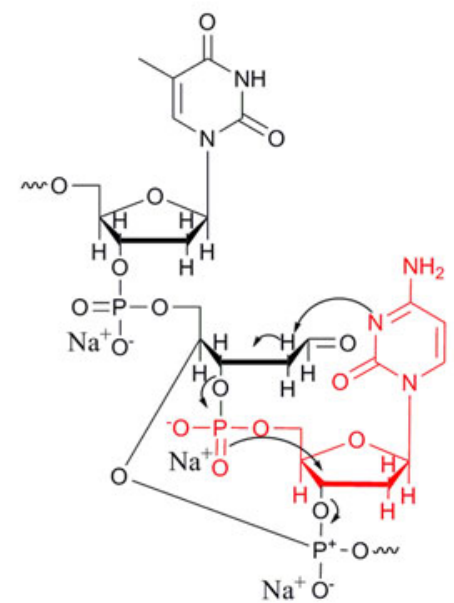

$[\mathbf{M}-\mathbf{B}]^{+}$

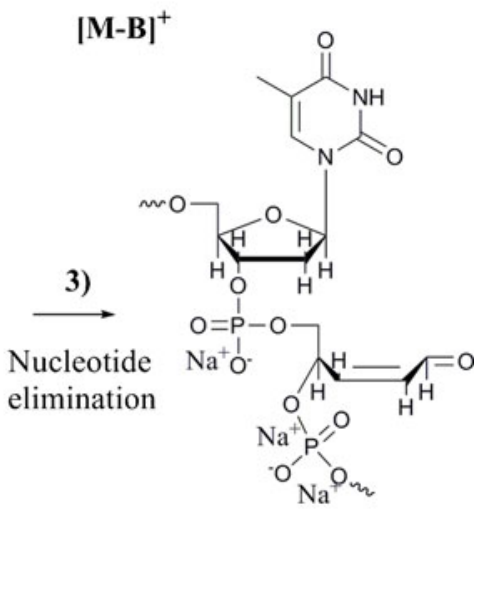

$\left(\mathrm{R}-\mathrm{H}_{2} \mathrm{O}\right) \mathrm{dT}_{4}$

Figure 6. Proposed mechanism for the elimination of a central nucleobase and nucleotide and a connection between the terminal $T$ chains, forming the fragment $\left(R-H_{2} O\right) d T_{4}$. The protonated high PA base is eliminated as a neutral fragment and the charge remains at its sugar moiety. A nucleophilic attack from a non-consecutive phosphodiester group to the 4 '-carbon center of the positively charged sugar forms the terminal TT connection. This leads to sugar ring opening and formation of a $1^{\prime}-\mathrm{C}=\mathrm{O}$ carbonyl, which increases the acidic character of the $2^{\prime}-\mathrm{C}-\mathrm{H}$. The second, high PA base removes the 2'-C-H proton and the 3'-C-O bond at the initial base elimination site is dissociated through an E2 mechanism. The released phosphate forms a 5'-3' cyclic phosphate to dissociate the next $3^{\prime}-\mathrm{C}-\mathrm{O}$ bond and a neutral nucleotide is eliminated to form $\left(\mathrm{R}-\mathrm{H}_{2} \mathrm{O}\right) \mathrm{dT} \mathrm{T}_{4}$. Here, the $3 \mathrm{~N}$ of the cytosine is shown as the base for proton removal 
diester group on the respective positively charged sugar moiety follows. This leads to ring opening of the sugar and an aldehyde formation at the 1 '-C, which in turn results in an acidic character of its $\beta$-hydrogen at the $2^{\prime}-\mathrm{C}$. The second high PA base then removes the acidic $\beta$-hydrogen, $2^{\prime}-\mathrm{C}-\mathrm{H}$, and induces an anti E2 elimination dissociating the $3{ }^{\prime}-\mathrm{C}-\mathrm{O}$ bond to the sugar that was previously connected to the nucleotide carrying the first high PA base. The released phosphate further forms a 5'-3'-cyclic phosphate and the nucleotide $3^{\prime}-\mathrm{C}-\mathrm{O}$ bond is dissociated. The nucleotide containing the second high PA base is thus eliminated as a neutral fragment, leading to a positively charged ion containing the terminal thymidine groups and the sugar (less one water) originally connected to the first high PA base. This mechanism is concordant with the initial loss of the first high PA base and rationalizes the necessity of the second high PA base to enable the $\left(\mathrm{R}-\mathrm{H}_{2} \mathrm{O}\right) \mathrm{dT}_{4}$ formation.

\section{Conclusions}

For short, single stranded, protonated oligonucleotides containing terminal thymidine groups we have shown that central nucleotide deletion and recombination of the terminal nucleotides can become the dominating fragmentation channel in the gas phase upon replacing the exchangeable protons with sodium ions. We find that the first step in this intriguing fragmentation is an elimination of a protonated high PA base, but also a second adjacent high PA base is required to enable this channel to proceed beyond the first base loss. It is also clear that the recombination of the terminal nucleotides proceeds between the thymidines next to the departing high PA bases and that the reactive conformation necessary for sufficient proximity of these, requires folding of the ONTs. This fold is most likely achieved (stabilized) upon $\mathrm{H}^{+} / \mathrm{Na}^{+}$exchange. To our knowledge, this and a previous study by our group [13], are the first reported experimentally verified central eliminations of nucleotides from single stranded oligonucleotides in the gas phase. In biological systems, however, where the secondary structure and DNA association with other biologically relevant molecules such as proteins is very important [1-3], central nucleotide deletion is well known. It is also well known that the aqueous environment and DNA ion adduct formation influences nucleobase stacking, and more generally the interactions between individual nucleotides. In many cases, gas phase studies can provide information that is not readily attained in the condensed phase, but the relevance of such studies for biological systems has often been disputed. It is thus very desirable to be able to mimic the conditions found in biological systems in the gas phase. Preparation of well-defined secondary structures in the gas phase would be an important step in this direction. In the current study, it has been shown that secondary structures can define the reactivity of ONTs in the gas phase and lead to reactions similar to those observed in biological systems. It has also been shown that sodium ions (and other ions) in combination with defined sequences might be used to produce well-defined secondary structures. If such structures could be produced in a controlled way, and would be characterized with ion mobility measurements in combination with molecular dynamics calculations as has been done before $[4,20]$, they would provide a very valuable model systems for a wealth of gas-phase studies. Also, infrared multiphoton dissociation mass spectrometry is a promising tool for characterizing secondary DNA structures in the gas phase [21].

\section{Acknowledgment}

The authors acknowledge support for this work by the Icelandic Centre for Research (RANNIS) and by the University of Iceland Research Fund. HDF acknowledges a PhD grant from the Eimskip University Fund. This work was conducted within the framework of the COST Actions CM0601 (ECCL) and MP1002 (Nano-IBCT).

\section{References}

1. Singleton, C.K.: Effects of salts, temperature, and stem length on supercoil-induced formation of cruciforms. J. Biol. Chem. 258, 76617668 (1983)

2. Singleton, C.K., Wells, R.D.: Relationship between superhelical density and cruciform formation in plasmid-pvH51. J. Biol. Chem. 257, 62926295 (1982)

3. Noirot, P., Bargonetti, J., Novick, R.P.: Initiation of rolling-circle replication in pT181 plasmid-initiator protein enhances cruciform extrusion at the origin. Proc. Natl. Acad. Sci. U. S. A. 87, 8560-8564 (1990)

4. Baker, E.S., Bernstein, S.L., Bowers, M.T.: Structural characterization of G-quadruplexes in deoxyguanosine clusters using ion mobility mass spectrometry. J. Am. Soc. Mass Spectrom. 16, 989-997 (2005)

5. Baker, E.S., Gidden, J., Ferzoco, A., Bowers, M.T.: Sodium stabilization of dinucleotide multiplexes in the gas phase. Phys. Chem. Chem. Phys. 6, 2786-2795 (2004)

6. Basu, S., Rambo, R.P., Strauss-Soukup, J., Cate, J.H., Ferre-D'Amare, A.R., Strobel, S.A., Doudna, J.A.: A specific monovalent metal ion integral to the AA platform of the RNA tetraloop receptor. Nat. Struct. Biol. 5, 986-992 (1998)

7. Parkinson, G.N., Lee, M.P.H., Neidle, S.: Crystal structure of parallel quadruplexes from human telomeric DNA. Nature 417, 876-880 (2002)

8. Hong, E.S., Yoon, H.J., Kim, B., Yim, Y.H., So, H.Y., Shin, S.K.: Mass spectrometric studies of alkali metal ion binding on thrombinbinding aptamer DNA. J. Am. Soc. Mass Spectrom. 21, 1245-1255 (2010)

9. Black, C.B., Huang, H.W., Cowan, J.A.: Biological coordination chemistry of magnesium, sodium, and potassium-ions. Protein and nucleotide-binding sites. Coord. Chem. Rev. 135, 165-202 (1994)

10. Martínez, J.M., Elmroth, S.K.C., Kloo, L.: Influence of sodium ions on the dynamics and structure of single-stranded DNA oligomers: A molecular dynamics study. J. Am. Soc. 123, 12279-12289 (2001)

11. Remko, M., Van Duijnen, P.T., von der Lieth, C.W.: Structure and stability of $\mathrm{Li}(\mathrm{I})$ and $\mathrm{Na}(\mathrm{I})$-carboxylate, sulfate, and phosphate complexes. THEOCHEM J. Mol. Struct. 814, 119-125 (2007)

12. Fales, B.S., Fujamade, N.O., Nei, Y.W., Oomens, J., Rodgers, M.T.: Infrared multiple photon dissociation action spectroscopy and theoretical studies of diethyl phosphate complexes: Effects of protonation and sodium cationization on structure. J. Am. Soc. Mass Spectrom. 22, 81$92(2011)$

13. Flosadóttir, H.D., Stano, M., Ingólfsson, O.: Sodium controlled selective reactivity of protonated deoxy-oligonucleotides in the gas phase. J. Am. Soc. Mass Spectrom. 20, 689-696 (2009)

14. Stano, M., Flosadóttir, H.D., Ingólfsson, O.: Effective quenching of fragment formation in negative ion oligonucleotide matrix-assisted laser desorption/ionization mass spectrometry through sodium adduct formation. Rapid Commun. Mass Spectrom. 20, 3498-3502 (2006) 
15. Solomon, E., Borrow, J., Goddard, A.D.: Chromosome aberrations and cancer. Science 254, 1153 (1991)

16. Barhate, N., Cekan, P., Massey, A.P., Sigurdsson, S.T.: A nucleoside that contains a rigid nitroxide spin label: A fluorophore in disguise. Angew. Chem. Int. Ed. 46, 2655-2658 (2007)

17. McLuckey, S.A., Vanberkel, G.J., Glish, G.L.: Tandem mass-spectrometry of small, multiply charged oligonucleotides. J. Am. Soc. Mass Spectrom. 3, 60-70 (1992)

18. Greco, F., Liguori, A., Sindona, G., Uccella, N.: Gas-phase proton affinity of deoxyribonucleosides and related nucleobases by fast atom bombardment tandem mass spectrometry. J. Am. Soc. Mass Spectrom. 112, 9092-9096 (1990)

19. Cooks, R.G., Patrick, J.S., Kotiaho, T., McLuckey, S.A.: Thermochemical determinations by the kinetic method. Mass Spectrom. Rev. 13, 287-339 (1994)

20. Gidden, J., Baker, E.S., Ferzoco, A., Bowers, M.T.: Structural motifs of DNA complexes in the gas phase. Int. J. Mass Spectrom. 240, 183-193 (2005)

21. Yuan, G., Zhang, Q., Zhou, J., Li, H.H.: Mass spectrometry of G-quadruplex DNA: Formation, recognition, property, conversion, and conformation. Mass Spectrom. Rev. 30, 1121-1142 (2011) 